\title{
Demographic Equality of Access to Cognitive Behavioural Treatment for Early Psychosis in Community Mental Healthcare in Greater London
}

\author{
By Pawel D. Mankiewicz, Jordan Reid ${ }^{ \pm} \&$ Eleanor Anne Hughes ${ }^{*}$
}

\begin{abstract}
Individuals experiencing psychosis have been described as one of the most stigmatised, disadvantaged and socially excluded groups in modern societies. Despite the development of evidence-based psychological treatments for psychosis, the access to such intervention remains debateable. In metropolitan areas, where clinical caseloads of mental health services should reflect the ethnic diversity of local populations, equal provision of recommended therapies might be further confounded, while empirical literature investigating this appears limited. The present study inspects equality of access to cognitive behavioural therapy for psychosis across four specialist healthcare teams located in Greater London. Subsequent acceptance and participation in treatment are also examined. A retrospective analysis of electronic medical records of 244 patients was conducted using binary logistic regression and multiple linear regression. Participants were shown to have received treatment offers equally across demographic variables. Likewise, once the treatment commenced, engagement was equally distributed. However, despite having equal access to the offered treatment, patients of Black ethnic group seemed less likely to accept such offer, hence appeared disadvantaged in terms of treatment provision. This study demonstrates that, in multicultural communities, equal access to core interventions for psychosis is achievable, however does not on its own merits ensure equitable treatment provision. Thus mental health services need to proactively focus on specific ethnic groups requiring further efforts to increase treatment uptake.
\end{abstract}

Keywords: mental health, psychosis, cognitive behavioural treatment, diversity, equal access to healthcare

\section{Introduction}

A growing awareness of inequalities affecting individuals with complex mental health needs, such as psychosis, in terms of both social participation and accessing evidence-based psychological treatments, has been a subject of peerreviewed publications in recent years. For instance, difficulties in entering adequate employment opportunities among psychiatric outpatients were illustrated by Fernandes et al. (2017), despite such opportunities being an important therapeutic resource that supports their psychosocial recovery, ensures interpersonal

\footnotetext{
*Clinical Teaching Fellow, Research Department of Clinical, Educational and Health Psychology, University College London, \& Senior Clinical Psychologist, National Health Service, NorthEast London NHS Foundation Trust, UK.

${ }^{ \pm}$Trainee Clinical Psychologist, National Health Service, North-East London NHS Foundation Trust, UK.

${ }^{\ddagger}$ Assistant Psychologist, National Health Service, North-East London NHS Foundation Trust, UK.
} 
connectedness, maintains social inclusion, drives rehabilitative experiences, enhances wellbeing, and dignifies those diagnosed with a mental health disorder.

Likewise, in the current context of rapidly aging population in numerous countries around the world, the inequalities in provision of mental health services to older individuals with psychiatric diagnoses were described by Rajamanickam et al. (2019). As shown by Newson et al. (2020), a widespread lack of homogeneity in the assessment of individuals across a range of mental health needs hinders identification of differential diagnoses, disrupts the understanding of social and environmental factors contributing to the maintenance of emotional distress, and ultimately leads to inconsistencies in the provision of recommended treatments.

This paper reviews the topical literature and outlines inequalities that are faced by individuals with one of the most stigmatising psychiatric diagnoses, that is psychosis, in both social and institutional contexts. The article reflects on socially inclusive advancements in national policies effecting gradual transformation in British mental healthcare system, exemplified by early intervention services. The central importance of equal, inclusive, and non-discriminatory provision of evidence-based psychological therapies is demonstrated and argued as a starting point toward wider societal changes. Finally, as a gap in existing literature investigating equal access to mental healthcare is identified, a quantitative study examining equality of access to psychological treatment for psychosis among ethnically diverse clinical population of Greater London is reported, implications of which are then indicated.

\section{Social Exclusion in Psychosis}

Clinical presentation of psychosis can be characterized according to international diagnostic criteria, which typically describe a number of symptom domains, including auditory hallucinations (hearing voices), persecutory or paranoid delusions (erroneous, fixed, and distressing beliefs that are not amenable in the light of conflicting evidence), disorganized thought and speech, disorganized behaviour, and negative symptoms, that is diminished emotional expression and avolition (Heckers et al. 2013). The experiences of being subjected to a form of discrimination and recurrent exposure to prejudice and social adversity have been identified among the factors contributing to the onset, maintenance and exacerbation of symptoms of psychosis (Pearce et al. 2019). Existing studies demonstrate that stigma and prejudice toward individuals with psychosis prevent social inclusion, postpone help-seeking behaviour, and delay psychosocial recovery (Baba et al. 2017). People with experiences of psychosis report feeling trapped within the discouraging context of social inequality, which in their opinion often results in collective dismissal of their resilience and potential productivity, and contributes to their stigmatisation, discrimination and exclusion (Campbell 2007).

As argued by Lingwood (2006), people with complex mental health difficulties, such as psychosis, have been among the most excluded social groups, often experiencing widespread stigma and discrimination. Such widespread marginalisation of people with mental ill-health, as proposed by Social Exclusion Unit (SEU 2004), might have been maintained and exacerbated by negative 
assumptions shared by the public about the capabilities of individuals with psychiatric diagnoses, contributing to the inhibition of their recovery. In the UK, The Royal College of Psychiatrists (RCP 2009) recognised that low expectations as to what people with mental ill-health are capable of achieving are prevalent in society, which in turn perpetuate self-stigmatisation where affected individuals internalise and act on the low expectations that others have of them.

\section{Discrimination and Psychosis}

A recent systematic review of quantitative studies conducted by Pearce et al. (2019) demonstrated clear links between the experiences of discrimination based on ethnicity and higher rates of psychosis. In Western Europe, incidence of psychosis appears considerably higher among immigrants and ethnic minorities. For instance, in the UK the risk of experiencing psychosis is significantly higher for Africans and Caribbeans, while in the Netherlands the prevalence of psychosis seems elevated mainly among the immigrants from Northern Africa (Fearon et al. 2006, Veling et al. 2007). Such discrepancies appear explainable by the social contexts in which the minorities exist, where discrimination based on ethnicity and race appears to have a particularly adverse effect on mental health of targeted individuals (Boydell et al. 2001, Cantor-Graae and Selten 2005).

Empirical literature demonstrates a clear statistical association between experiences of discrimination and the onset (Janssen et al. 2003) and prevalence (Karlsen et al. 2005) of psychosis. The outcomes of an epidemiological study led by Veling et al. (2007) suggested that, rather than the experience of immigration, solely belonging to an ethnic minority group reporting a high degree of discrimination is by itself a risk factor for the development of psychosis. Such finding appears consistent with other studies, which demonstrate that migration itself does not account for increased risk of psychosis, as the incidence rates for first- and second-generation immigrants seem on average comparable, while ethnic minorities who have never experienced migration are also at greater risk (Bourque et al. 2011, Bresnahan et al. 2007).

\section{Social Inclusion in Mental Health Policies and Medical Services}

In the UK, national policies recognise that an instigation of socially inclusive attitudes toward individuals with psychiatric diagnoses must begin with the transformation of frontline healthcare. For instance, Department of Health (DOH 2007) recommended that mental health professionals must acknowledge their clients' personal, social, cultural and spiritual needs, and ensure provision of evidence-based treatment to all service users equally and equitably. Also, the National Social Inclusion Programme (2009) argued that one of key objectives for socially inclusive practice in mental health is to promote a meaningful participation in community life and support restoration of personal agency.

Mental health services in the UK are now required to demonstrate their socially inclusive function and provide interventions indiscriminately to all individuals in need of such support (DOH 2007). An increasing number of 
specialist mental health services for individuals with psychosis appear to have adopted the recommendations. Such services promote deinstitutionalisation, prioritise provision of psychological therapies, employ assertive outreach strategies to engage individuals in the offered treatment, have a shared goal of facilitating independence, and enable functional networks of support. Successful provision of a high-relational supportive service for individuals with complex mental ill-health, including psychosis, was illustrated by Davies et al. (2012). The authors reported significant gains in the areas of interpersonal integration, independence, and exercised choice among the service users. In addition, despite the continuous presence of clinical symptoms, an improvement in individual overall wellbeing was observed. Also, since employment rates among people with severe mental health needs in the UK were estimated as 3.4\%, Dominy and Hayward-Butcher (2012) reported a significant increase in subjective quality of life among service users following their re-employment.

\section{Early Intervention Healthcare for Psychosis}

Informed by socially inclusive policies, Early Intervention in Psychosis (EIP) teams are designated sectors of the National Health Service (NHS) commissioned to provide specialist multidisciplinary treatment to all individuals diagnosed with First Episode Psychosis (FEP). First episodes may occur at any time in life, however the onset usually takes place during late adolescence or early adulthood, with incidence rates estimated at 86 per 100,000 for those aged 15 to 29 and 46 per 100,000 for those aged 30 to 59 (Simon et al. 2017).

FEP can greatly impact an individual. A systematic review by Marshall et al. (2005) found convincing evidence of relationship between the duration of untreated psychosis (DUP) and negative overall functioning outcomes. There are also significant public health costs. National Institute for Health and Care Excellence (NICE 2016) estimated $£ 11.8$ billion a year is lost to reduced productivity, costs to carers, and direct health expenditure. This highlights a need for timely and effective care to improve prognosis for the individual as well as costs to the public.

Since their inception in 1999, EIP services have grown in number and there are now over 150 teams in the UK (McDaid et al. 2016). EIPs aim to reduce the DUP, thereby intending to improve prognosis. As such, standards have been set to ensure treatment is provided in a timely and equal manner, with NICE (2016) setting a target of 50\% of people experiencing FEP getting access to treatment within two weeks. Hansen et al. (2018), in their meta-synthesis of qualitative literature, explored how individuals diagnosed with FEP experienced their involvement with EIP services. The authors reported that EIP clients appraised receiving respectful treatment from team members, emphasising collaboration and social involvement, and accentuating personal agency, as crucial factors contributing to achieving a considerable progress in their recovery and social reengagement. 


\section{Provision of Psychological Therapies for Psychosis}

Centrality of psychological therapies in planning and delivering mental healthcare was emphasised by the British Psychological Society (2007), while the critical role of equal access to psychological treatment across the entire spectrum of severity of mental ill-health was argued by the SEU (2004) as key in reducing stigmatisation and social exclusion of those with psychiatric diagnoses. Consequently, NICE (2014) published its clinical guideline for psychosis, in which evidence-based psychological interventions were recommended as a principal treatment for all individuals with such needs. In particular, cognitive behavioural therapy for psychosis (CBTp) is now regarded as a core evidence-based psychological treatment for individuals experiencing psychosis and is widely implemented in specialist mental healthcare (NICE 2016). CBTp aims to build functional strategies to cope with symptoms of psychosis, such as voices and delusional beliefs, and reduce associated emotional distress. Therapeutic process is facilitated through a repertoire of interventions utilised collaboratively with service users to restructure their interpretation of anomalous experiences, reduce anxiety, overcome withdrawal, and facilitate gradual social reengagement (Mankiewicz 2013). Additionally, CBTp has been shown to help individuals in developing their personal strengths, increasing their sense of agency, and overcoming social exclusion through gradual exposure to participatory interaction with others (Mankiewicz 2019b).

Effectiveness of CBTp has been supported with affirmative outcomes of single subject design studies (e.g., Mankiewicz and Turner 2014, Mankiewicz 2019a), randomised control trials (e.g., Farhall et al. 2009), and comprehensive meta-analyses (e.g., Hazell et al. 2016). A single-blinded RCT conducted among participants who had chosen not to take antipsychotic medication (Morrison et al. 2014) produced further encouraging evidence.

Qualitative research provides additional justification for ensuring homogenous provision of CBTp. Particularly in FEP, CBTp participants report to value building practical everyday life skills, overcoming social exclusion, reengaging interpersonally, restructuring unhelpful beliefs, reducing emotional distress, and achieving an overall sense of relief and unburdening, as key factors in their psychosocial recovery (Mankiewicz et al. 2018). Interestingly, clinical recovery, that is alleviation of symptomatology, was not reflected on as having central importance in achieving desired therapeutic change. Indeed, the key role of regaining personal agency and augmenting social participation in psychosis has been increasingly recognised in clinical literature (Mankiewicz 2015).

The importance of achieving equal provision of CBTp as core treatment in early psychosis may also be justified within the context of clients' satisfaction rates, which approximate $96 \%$, while acceptability levels are rated as excellent and unaffected by demographics (Lawlor et al. 2017, Miles et al. 2007). These seem in marked contrast to pharmacological interventions with antipsychotic medication. As demonstrated in a randomised study by Liebermann et al. (2005), circa $60 \%$ of people with psychosis were unresponsive to medication, while the treatment discontinuation rate due to inefficacy or intolerable side effects approximated 
$74 \%$, indicating that merely $26 \%$ of psychiatric patients were somehow satisfied with their use of prescribed antipsychotics.

\section{Inequalities in Accessing and Engaging with CBTp and EIP}

Despite national guidelines, studies reviewed by Berry and Haddock (2008) revealed that CBTp was not routinely available to NHS service users. In fact, on average only $46 \%$ of those with psychosis in specialist mental healthcare were offered CBTp. The figures ranged from $20 \%$ to $96 \%$, suggesting wide variation across the services. It was also found that those with more acute, complex and severe presentations of psychosis appeared particularly disadvantaged in accessing evidence-based psychological treatment. As shown by Mankiewicz and Turner (2012), while CBTp was found to be delivered to $30.7 \%$ of the referred clients across the researched services, the intervention was accessed more often in community-based clinics, where $19.8 \%$ of the referred clients (36\% of those who actually attended appointments) received CBTp, in comparison with $6.9 \%$ in rehabilitation wards (12.7\% controlled for attendance) and $3.9 \%$ in acute inpatient settings ( $7.3 \%$ controlled for attendance).

Berry and Haddock (2008) argued that the main barriers to achieving equality in the implementation of clinical guidelines involved factors associated with organisations, clinicians, and patients. Organisational barriers included predominant focus on monitoring medication and managing risks and crises, in expense of provision of psychological therapies. Also, therapists did not seem adequately trained in CBTp or exhibited resistance due to unsubstantiated presumptions that psychosis was too complex to respond to such intervention. Likewise, as demonstrated by Mankiewicz et al. (2018), EIP service users held pre-existing unhelpful assumptions about psychological treatment, hence would either decline the offer or postpone help-seeking in the first place.

Age appears to be another factor contributing to the disparity in accessing CBTp. Many young people with mental health problems seem to delay seeking help from professionals (Slade et al. 2009). One reason for this might be negative expectations of therapy, which may act as a major barrier to achieving progress in recovery. Pre-existing negative attitudes toward receiving mental health treatment were shown to be associated with lower help-seeking, poorer engagement, and diminished recovery, whereas positive expectations were often linked with higher rates of improvement in psychosocial functioning and enhanced wellbeing (Dew and Bickman 2005).

NICE (2014) standards require mental health services to ensure that equality in provision of psychological treatment for psychosis is maintained and monitored institutionally. However, a national audit conducted by RCP (2019) found considerable variation between EIP services in delivering psychological interventions. Research has linked premature termination of early treatment of psychosis to negative longer term outcomes, such as poorer prognosis and increased likelihood of hospitalisation (Stewart 2013). Also, some studies suggested that uptake and engagement with treatment for FEP is far more relevant in determining outcomes than treatment efficacy (Gaynor et al. 2014). To improve 
treatment delivery, it is therefore vital for services to understand which factors may be relevant to disengagement from psychological interventions.

Demographic and clinical variables have been shown to impact on clients' engagement with EIP services. In their systematic review of literature, Nose et al. (2003) found that younger age and male gender were associated with poorer treatment compliance. Poorer engagement has also been shown among Black ethnic group. For instance, Anderson et al. (2013) found that patients of Black ethnicity were more likely to disengage from a two-year intervention for psychosis, when compared to their White counterparts. Additionally, faith and religion also appear to be associated with lower engagement with pharmacological and psychological treatments. A review by Maura and Weisman (2017) indicated that individuals who reported religious or spiritual beliefs were less likely to engage with mental health services and instead were more inclined to seek out spiritual or religious alternatives to medical input.

For clinical factors, substance misuse has been shown to contribute to disengagement from services. Conus et al. (2010) found that service users with FEP and substance misuse disorder were significantly more likely to disengage from treatment when compared to those without dual diagnoses. Furthermore, family involvement has been demonstrated to improve engagement with services. Stowkowy et al. (2012) found that having a family member involved in treatment was associated with longer periods of engagement with services. As indicated by the authors, $18 \%$ of people who disengaged in under six months had family members involved in their care, comparing to $76 \%$ of people who remained in treatment for 30-36 months.

It seems also likely that prior hospital admissions are predictive of poorer engagement with CBTp. A history of mental health hospitalisations has been shown to be associated with poorer outcomes, such as higher symptom severity and increased likelihood of further inpatient admissions (Wade et al. 2006, Addington et al. 2010). Jaeger et al. (2013) found that patients who had been involuntarily admitted to mental health wards reported lower intervention adherence and were more likely to feel coerced into treatment upon discharge. Finally, the aforementioned DUP appears to predict hospitalisations (Wade et al. 2006) as well as poorer engagement with CBTp (Alvarez-Jimenez et al. 2009).

\section{Methods}

The current study seeks to develop a comprehensive understanding of the degree to which the socially inclusive national policies recommending equal provision of evidence-based psychological therapies, i.e., CBTp, to all individuals diagnosed with psychosis, are being implemented in frontline mental health services in the UK, thus modelling the culture of inclusion, equality and diversity in the delivery of modern mental healthcare. 


\section{Study Context}

Due to the leading and culture-shaping role of early intervention services, which are sited in the forefront of British mental healthcare, and ethnically diverse population of Greater London, the study was conducted in four London-based EIP teams delivering NICE-concordant multidisciplinary interventions. The richness of diverse communities in London has long been reflected in academic literature and was described by Sturgis et al. (2014) as "the most ethnically diverse conurbation on the planet" (p. 1286), hence appeared a particularly suitable location to contextualise the current study.

Approval of the project was granted by clinical audit department of the NHS Trust hosting the study. Ethical considerations focused predominantly on the maintenance of anonymity of data and safeguarding confidentiality of clinical files, which had been obtained from electronic records department of the Trust. Strict data protection guidance was followed in all stages of the auditing procedure and no personally identifiable information was gathered or recorded in the process of data collection.

\section{Study Aims}

In accordance with clinical guidance (NICE 2014, 2016) CBTp should be offered to all individuals with FEP who come to the attention of mental healthcare, while the provision of psychological interventions, and equality of access across different ethnic groups, should be monitored and reviewed. Hence, the overall objective of the present study was to investigate the compliance with national guidelines in selected services collectively. The study investigated if demographic variables of gender, ethnicity, religion, age, and a set of clinical factors, such as substance use, history of inpatient admission, and family involvement, impacted on the equality of access to CBTp among the diverse clinical population of service users with FEP. Distribution of uptake and engagement with treatment was also examined. Thus, the equality of treatment provision was investigated at the following three service delivery stages: receiving an offer of treatment (access), accepting treatment offer (uptake), and commencing and participating in treatment (engagement).

\section{Sample}

The sample consisted of 244 service users across four London-based EIP teams (team $1 \mathrm{n}=41$; team $2 \mathrm{n}=72$; team $3 \mathrm{n}=65$; and team $4 \mathrm{n}=66$ ) who had been referred to the services in the year of 2018. The researched EIP teams provided multidisciplinary support to individuals with FEP across the lifespan, as the aforementioned national guidance necessitated such intervention to be offered to all patients with experiences of psychosis. Hence, no service users were excluded from data collection and analysis. The participants were between 15 and 64 years of age, with mean age of 31.9 and median of 28.7. Descriptive statistics outlining the sample diversity are presented in Table 1. 
Table 1. Demographic Variables: Descriptive Statistics $(n=244)$

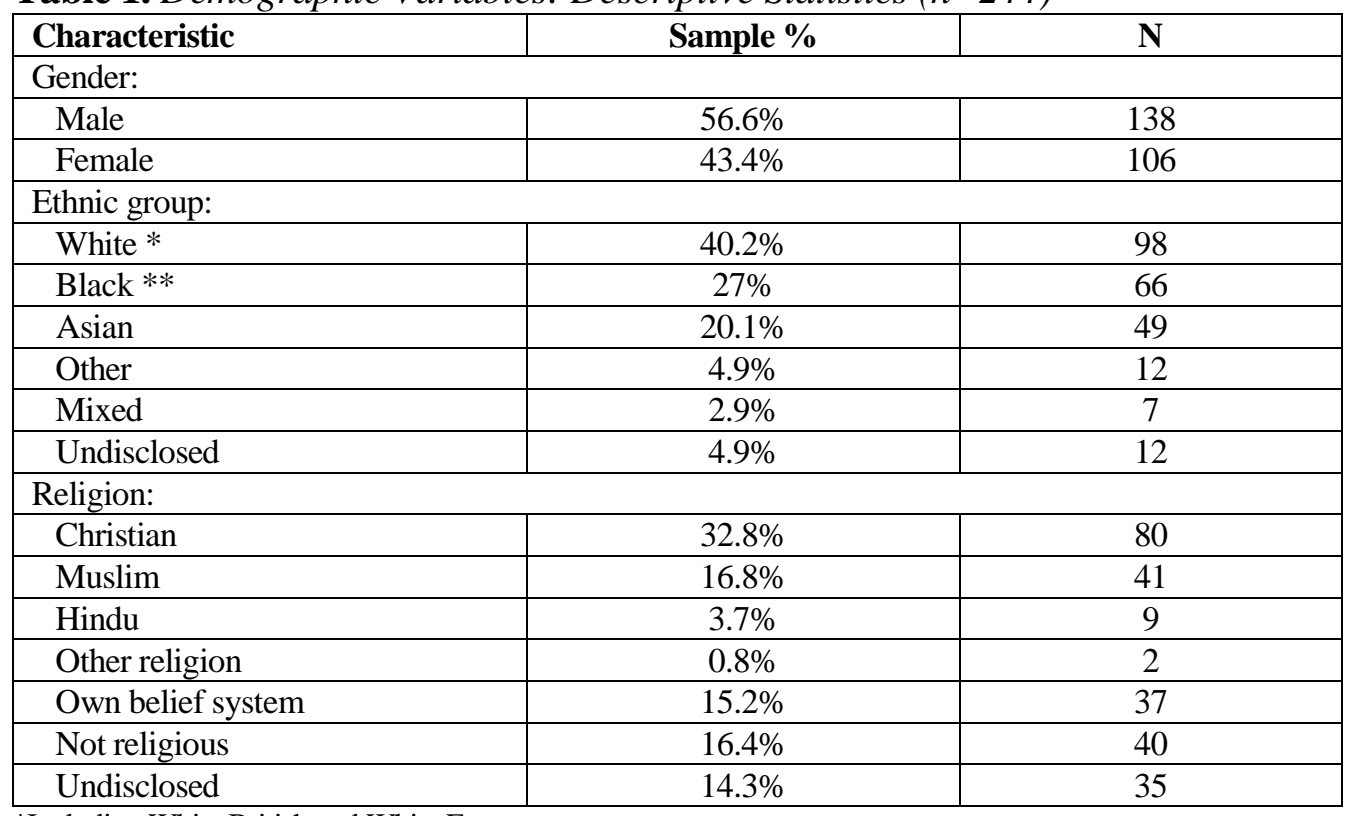

*Including White British and White European.

**Including Black British, Black African and Black Caribbean.

\section{Procedure}

The study employed a statistically rigorous retrospective analysis of electronic health records for all service users referred to the researched mental healthcare sites throughout the calendar year of 2018. Relevant data was counted until mid2019 to allow sufficient time for treatment offers to be made and accepted, and for CBTp intervention to commence.

\section{Data Analysis}

Statistical analyses were run on the Statistical Package for the Social Sciences (SPSS) software (IBM Corp 2017). Since each of the investigated treatment delivery phases, that is treatment offer (access), treatment acceptance (uptake), and participation in treatment (engagement), necessitated a unique approach, these were analysed autonomously.

At first, for treatment offers, a binary logistic regression was conducted, assessing the odds that a particular service user group would be offered, thus have access to, CBTp. No data was excluded from this analysis, as all participants would have been offered, or not offered, such treatment. Groups that lacked a socially meaningful denotation, such as undisclosed ethnicity, were included in the model to increase the power of statistical analysis, however not interpreted. For treatment uptake, a binary logistic regression was conducted, demonstrating the odds that a particular service user group would accept the treatment offer. Those participants that had not been offered CBTp were not included. Finally, a multiple linear regression was conducted, to see if membership of a client group predicts a number of attended treatment sessions, thus indicating the length of engagement. 
Only those participants that had accepted an offer of CBTp were included. For each of the above analyses, due to a large number of tested variables inflating the possibility of type-1-error, the Bonferroni correction was applied in order to reduce the likelihood of false positives.

Service users did not need to physically attend the clinic in order to be made an offer of the recommended treatment. The investigated services implemented an assertive outreach model of work (Mankiewicz and Turner 2012), in which clinicians were able to visit the patients at their homes or other mutually agreed locations to conduct assessments and provide support. Likewise, the treatment delivery did not necessitate the patients' ability to commute to the clinic. Some service users preferred to be seen at home or other mutually agreed sites to facilitate the treatment delivery. Such assertive outreach approach aimed to promote inclusion and provision of treatment to those service users who could not, or for some reasons preferred not to, attend their appointments in the clinic.

\section{Results}

For brevity, the reported findings will focus on the discussion of statistically significant results. If not mentioned below, non-significance of findings should be assumed.

\section{Offer of CBTp (Access)}

Results of the binary logistic regression indicated that there was a significant association between male gender and CBTp offers $(\chi 2(1)=6.42, p=0.011)$ indicating that males might have been less likely to be offered CBTp treatment. This association was subsequently shown as non-significant after Bonferroni correction. There was also a significant association between mixed race ethnicity and being offered CBTp $(\chi 2(1)=4.89, \mathrm{p}=0.027)$ suggesting that there might have been a lower likelihood for mixed-race individuals to be offered CBTp. Likewise, this was not significant after Bonferroni correction. Details of statistical analyses for treatment offers are illustrated in Table 2. 
Table 2. Odds Ratio Estimates for All Demographic and Clinical Variables Predicting CBTp Treatment Offer $(n=244)$

\begin{tabular}{|c|c|c|}
\hline & \multirow{2}{*}{\multicolumn{2}{|c|}{ 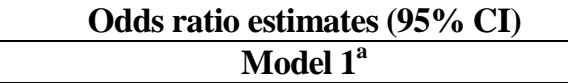 }} \\
\hline & & \\
\hline Age & 1.001 & $(0.97-1.03)$ \\
\hline \multicolumn{3}{|l|}{ Sex } \\
\hline Female & 1.00 & (ref) \\
\hline Male & .39 & $(0.18-0.81)^{*}$ \\
\hline \multicolumn{3}{|l|}{ Ethnic group } \\
\hline White & 1.00 & (ref) \\
\hline Black & 0.97 & $(0.42-2.25)$ \\
\hline Asian & 1.58 & $(0.41-6.03)$ \\
\hline Other & 3.12 & $(0.35-27.9)$ \\
\hline Mixed & 0.15 & $(0.03-0.81)^{*}$ \\
\hline Ethnicity undisclosed & 1.78 & $(0.37-8.67)$ \\
\hline \multicolumn{3}{|l|}{ Religion } \\
\hline Christian & 1.00 & (ref) \\
\hline Muslim & 1.45 & $(0.37-5.67)$ \\
\hline Hindu & 0.46 & $(0.05-3.94)$ \\
\hline Other religion & 115758596.27 & $(0.00-0.00)$ \\
\hline Own belief system & 1.05 & $(0.36-3.04)$ \\
\hline Not religious & 0.92 & $(0.33-2.61)$ \\
\hline Religion undisclosed & 0.34 & $(0.12-0.97)$ \\
\hline \multicolumn{3}{|l|}{ Family involvement } \\
\hline No family involvement & 1.00 & (ref) \\
\hline Family involved & 1.16 & $(0.48-2.84)$ \\
\hline \multicolumn{3}{|l|}{ Substance use } \\
\hline No current substance use & 1.00 & (ref) \\
\hline Current substance use & 0.89 & $(0.31-2.58)$ \\
\hline \multicolumn{3}{|l|}{ Inpatient admission } \\
\hline No history of admission & 1.00 & (ref) \\
\hline History of admission & 0.68 & $(0.34-1.33)$ \\
\hline
\end{tabular}

*Non-significant after Bonferroni correction (.05/16=0.003).

${ }^{a}$ Model controlled for age, sex, ethnicity, religion, family involvement, substance use, and inpatient admission. ref=reference group.

\section{Acceptance of CBTp Offer (Uptake)}

As outlined in Table 3, the results of binary logistic regression indicated there was a significant association between age and accepting the offer of CBTp $(\chi 2(1)=4.93, p=0.026)$ indicating that, across the entire dataset, with increase in age participants might have been gradually less likely to accept an offer of CBTp. This result was subsequently shown as non-significant after Bonferroni correction was implemented. A significant association was also shown between Black ethnicity and accepting the offer of CBTp $(\chi 2(1)=9.54, \mathrm{p}=0.002)$ suggesting that individuals belonging to this particular ethnic group appeared less likely to accept the offer of psychological treatment when compared with their White counterparts. This result remained significant after Bonferroni correction. 
Table 3. Odds Ratio Estimates for All Demographic and Clinical Variables Predicting Acceptance of CBTp Treatment Offer $(n=244)$

\begin{tabular}{|c|c|c|}
\hline & \multirow{2}{*}{\multicolumn{2}{|c|}{$\frac{\text { Odds ratio estimates }(95 \% \text { CI) }}{\text { Model 1 }^{\mathrm{a}}}$}} \\
\hline & & \\
\hline Age & 0.96 & $(0.93-0.99)^{*}$ \\
\hline \multicolumn{3}{|l|}{ Sex } \\
\hline Female & 1.00 & (ref) \\
\hline Male & 0.47 & $(0.21-1.06)$ \\
\hline \multicolumn{3}{|l|}{ Ethnic group } \\
\hline White & 1.00 & (ref) \\
\hline Black & 0.19 & $(0.07-0.54)$ \\
\hline Asian & 0.29 & $(0.07-1.17)$ \\
\hline Other & 0.48 & $(0.07-3.09)$ \\
\hline Mixed & 0.13 & $(0.01-1.30)$ \\
\hline Ethnicity undisclosed & 0.13 & $(0.02-0.76)$ \\
\hline \multicolumn{3}{|l|}{ Religion } \\
\hline Christian & 1.00 & (ref) \\
\hline Muslim & 1.07 & $(0.27-4.21)$ \\
\hline Hindu & 0.80 & $(0.09-7.19)$ \\
\hline Other religion & 390507675.64 & $(0.00-0.00)$ \\
\hline Own belief system & 1.60 & $(0.40-6.45)$ \\
\hline Not religious & 0.75 & $(0.22-2.63)$ \\
\hline Religion undisclosed & 0.45 & $(0.12-1.77)$ \\
\hline \multicolumn{3}{|l|}{ Family Involvement } \\
\hline No family involvement & 1.00 & (ref) \\
\hline Family involved & 0.85 & $(0.31-2.33)$ \\
\hline \multicolumn{3}{|l|}{ Substance Use } \\
\hline No current substance use & 1.00 & (ref) \\
\hline Current substance use & 0.644 & $(0.20-2.07)$ \\
\hline \multicolumn{3}{|l|}{ Inpatient admission } \\
\hline No history of admission & 1.00 & (ref) \\
\hline History of admission & 0.76 & $(0.36-1.64)$ \\
\hline
\end{tabular}

*Non-significant after Bonferroni correction $(0.05 / 16=0.003)$.

${ }^{a}$ Model controlled for age, sex, ethnicity, religion, family involvement, substance use, and inpatient admission. ref=reference group.

\section{Engagement in CBTp (Participation)}

As shown in Table 4, no variables were significantly associated with engagement in the offered psychological treatment that had been taken up by the service users across the entire dataset, indicating equal participation in CBTp treatment once accepted and commenced. 
Table 4. Parameter Estimates and Confidence Interval Limits for All Demographic and Clinical Variables Predicting Engagement with CBTp Treatment for Entire Data set $(n=244)$

\begin{tabular}{|c|c|c|c|c|}
\hline & Estimate & $S E$ & $95 \% \mathrm{CI}$ & $\boldsymbol{P}$ \\
\hline Age & 0.039 & 0.048 & $(-0.06-0.14)$ & 0.418 \\
\hline \multicolumn{5}{|l|}{ Sex } \\
\hline Female & ref & & & \\
\hline Male & 0.441 & 1.187 & $(-1.91-2.79)$ & 0.711 \\
\hline \multicolumn{5}{|l|}{ Ethnic group } \\
\hline White & ref & & & \\
\hline Black & -1.442 & 1.530 & $(-4.47-1.59)$ & 0.348 \\
\hline Asian & -0.236 & 2.503 & $(-5.19-4.72)$ & 0.925 \\
\hline Other & 1.339 & 2.531 & $(-3.67-6.35)$ & 0.598 \\
\hline Mixed & 7.361 & 5.007 & $(-2.55-17.27)$ & 0.144 \\
\hline Ethnicity undisclosed & -1.230 & 3.639 & $(-8.43-5.97)$ & 0.736 \\
\hline \multicolumn{5}{|l|}{ Religion } \\
\hline Christian & ref & & & \\
\hline Muslim & -0.268 & 2.547 & $(-5.31-4.77)$ & 0.916 \\
\hline Hindu & -2.333 & 3.906 & $(-10.06-5.4)$ & 0.551 \\
\hline Other religion & -5.723 & 5.202 & $(-16.02-4.57)$ & 0.273 \\
\hline Own belief system & 0.219 & 1.761 & $(-3.27-3.70)$ & 0.901 \\
\hline Not religious & 0.414 & 1.731 & $(-3.01-3.84)$ & 0.811 \\
\hline Religion undisclosed & 0.588 & 2.343 & $(-4.05-5.23)$ & 0.802 \\
\hline \multicolumn{5}{|l|}{ Family involvement } \\
\hline No family involvement & ref & & & \\
\hline Family involved & 0.310 & 1.534 & $(-2.73-3.35)$ & 0.840 \\
\hline \multicolumn{5}{|l|}{ Substance use } \\
\hline No current substance use & ref & & & \\
\hline Current substance use & 1.127 & 2.002 & $(-2.83-5.09)$ & 0.574 \\
\hline \multicolumn{5}{|l|}{ Inpatient admission } \\
\hline No history of admission & ref & & & \\
\hline History of admission & 1.855 & 1.245 & $(-0.61-4.32)$ & 0.139 \\
\hline ref $=$ reference group & & & & \\
\hline
\end{tabular}

\section{Discussion}

The study has shown that offers of core psychological therapy for psychosis, i.e., CBTp, were made correspondingly across all investigated demographic variables, hence the service users with various ethnic backgrounds were able to access to the nationally recommended treatment equally. Such finding seems encouraging and demonstrates that equal provision of evidence-based treatment to a diverse, multi-ethnic population is, in principle, achievable.

A few disparities, however, were indicated in the initial analysis prior to the implementation of Bonferroni adjustments. For instance, males appeared less likely than females to have a recorded offer of CBTp. The literature may provide some understanding of why in some circumstance males may be less likely to be offered psychological therapy. Myhr et al. (2007) found that males scored lower on the scale of suitability for short-term cognitive therapy and were less likely to show a clinically significant improvement in symptoms and functioning. This may 
have an effect on therapists being less likely to offer therapy to those they do not believe will benefit, which in turn might perpetuate the trend of males having less advantageous access to psychological therapy, hence having lower opportunity to benefit from such treatment. Also, it was initially indicated that mixed-race service users appeared less likely than their White counterparts to have a recorded offer of CBTp. Academic literature on the subject of mixed-race patients and psychological therapies is limited. The use of mixed-race as a distinct category may lack reliability, as it encompasses a large number of various identities with divergent ethnic origins and cultural influences. Nonetheless, Milan and Keiley (2000) noted some difficulties mixed-race people may face above their mono-racial counterparts. The authors argued that biracial or biethnic youths were particularly vulnerable in terms of symptom internalisation, which might have resulted in lower likelihood to seek psychological help.

However, the above associations were subsequently demonstrated as statistically non-significant when Bonferroni method was applied, indicating the likelihood of type-1-error. As explained in statistical literature, Bonferroni correction is commonly utilised in multiple variables testing within the context of clinically embedded studies to control for type-1-error, that is incorrect rejection of null hypothesis (Bland and Altman 1995). The method is commonly used in social sciences for adjusting the significance levels of individual tests when multiple analyses are performed on the same datasets. The adjustment divides the nominal significance level by the number of tests being performed simultaneously to prevent the overall significance from exceeding the nominal level (Mundfrom et al. 2006). As argued by Sedgwick (2014), when multiple testing is subjected to Bonferroni correction and no significant differences are then seen, it is plausible to infer that the significant result observed initially was a type-1-error. Likewise, in multiple regression, conducting numerous separate tests would likely inflate the overall type-1-error rate for such analysis. Hence, as demonstrated by Mundfrom et al. (2006), using Bonferroni adjustment provides a dependable statistical protection against the inflated likelihood of false positives.

Nevertheless, if the aforementioned unhelpful preconceptions related to gender based unsuitability to treatment were identified in medical settings, these would ideally need to be openly discussed in clinical supervision and steps would need to be undertaken to encourage, govern and monitor equality in treatment offers across the genders. Likewise, if race related differences in treatment offers were identified in healthcare settings, immediate governance steps would need to be undertaken to ensure that all ethnic groups have equal access to recommended evidence based treatments. Clinical audit methodology (e.g., Mankiewicz and Turner 2012) would likely prove essential in achieving and monitoring such improvement in delivery of medical services.

Regarding the uptake of CBTp, the results suggested that treatment offers were accepted correspondingly across the majority of tested variables. Yet, the initial analysis indicated that for each year an individual aged they were marginally less likely to accept an offer of CBTp. The literature seems limited regarding age and acceptability of psychological therapies. One study examined the relationship between age and psychological treatments in FEP and found that 
older participants were inclined to respond better to CBTp and were actually easier to engage (Haddock et al. 2006). Although this finding may be at first seen as contrasting with the current results, considering the present study operationalised and tested the concepts of access, acceptance, and engagement separately, it is likely that the current methodology is comparatively more rigorous. As in previously tested variables for treatment offers, indicating equal access to CBTp, the age was demonstrated as statistically non-significant after Bonferroni adjustment was applied, indicating the presence of type-1-error.

Importantly, our analysis suggested that service users belonging to Black ethnic group appeared less likely than their White counterparts to accept an offer of CBTp across the entire dataset, which remained significant after Bonferroni correction. This result suggested some disparity in the actual commencement of the core treatment for FEP and indicated lack of equitability in the actual treatment provision, despite the identified equality in treatment access. The current finding seems interesting particularly when considered in the context of prevalence of psychosis in Black communities. An adult psychiatric morbidity survey conducted in the UK demonstrated that Black men were more likely than White males to experience psychosis (McManus et al. 2016). Also, the risk of such disorder in Black Caribbean communities was estimated to be approximately seven times higher comparing with White population (Fearon et al. 2006). Consequently, individuals from these groups appeared more likely than average to come to the attention of mental health services (Baker 2020). Thus, as demonstrated in the present study, although Black service users with FEP had equal access to recommended treatment, there was a discrepancy between the need for psychological therapy and the actual commencement of the offered intervention.

Ajnakina et al. (2017) suggested that ethnic minority clients were more likely to disengage from mental health services entirely, which might be linked with a higher level of self-perpetuated social disengagement in response to adverse life events that service users of Black ethnic group face in their communities, including criminal acts and police involvement, thus increasing the likelihood that acceptance of mental health treatment would not be subjectively prioritised. However, such supposition might be seen as placing responsibility entirely with the individuals of specific ethnicity and does not seem to explain the identified discrepancy from a systemic perspective. Interestingly, Ajnakina et al. (2017) also found higher rates of compulsory re-admissions and police involvement during admissions to psychiatric units among patients of Black African and Black Caribbean ethnicity compared with White British patients, which might indicate that individuals of Black ethnicity were potentially more likely to experience social disadvantages resulting in barriers to receiving treatment. Therefore, mental health services need to consider how these barriers could be better addressed.

Institutional obstacles to equitable provision of evidence based treatment for psychosis have long been identified in the topical literature. Studies reviewed by Berry and Haddock (2008) suggested that one of the main barriers to achieving equality in the implementation of clinical guidelines and provision of recommended treatment was related to factors associated with healthcare institutions. Organisational obstacles included predominant focus on managing 
risks and crises, often in expense of provision of psychological therapies. Also, therapists expressed resistance due to unsubstantiated presumptions concerning the provision of recommended interventions. Furthermore, as demonstrated by Mankiewicz and Turner (2012), CBTp provision rates appeared negatively associated with the severity of presentation of psychosis, with patients admitted to acute psychiatric wards being seemingly most disadvantaged in accessing the treatment. Hence, when such association is contextualised in the aforementioned higher compulsory inpatient admission trends among patients of Black ethnicity (Ajnakina et al. 2017), it appears even more important to acknowledge and proactively address the need to enhance CBTp treatment uptake rates among this vulnerable service user group.

Finally, the current study demonstrated that, once CBTp offer was accepted and treatment commenced, engagement with psychological therapy in early psychosis was equally distributed across all demographic variables. Such finding indicates compliance with national clinical guidance and is an encouraging result, illustrating equal participation in core psychological therapy for psychosis among multi-ethnic individuals with FEP.

\section{Conclusion}

An increasing number of mental health professionals undertake post-graduate training in CBTp. Their competencies are then utilised to augment the medical services' capacity to deliver the nationally recommended core treatment. Such increase in highly skilled workforce seems particularly relevant to early intervention teams. Mental health clinicians may choose to progress to leadership roles, in which they are responsible for overseeing the delivery of recommended intervention packages, including CBTp. This study demonstrates that, in multicultural communities, an equal access to core interventions for psychosis is achievable, however does not on its own merits ensure equitable treatment provision. The present study helps those in managerial positions in mental healthcare proactively target specific ethnic groups requiring further efforts to increase treatment uptake.

Overall, the study has shown that equal access to recommended psychological treatment among individuals with experiences of psychosis can be ensured across a range of demographic variables, including ethnic diversity, however further efforts need to be made to ensure equal treatment uptake among all vulnerable patient groups. Such results demonstrate that mental health services can play an important culture-shaping role, encouraging wider socially inclusive changes, improving attitudes toward one of the most disadvantaged, stigmatised and socially excluded groups in modern societies. As indicated by DaCosta (2020), progressive change in healthcare should be aligned with its strategic aims. As such, inclusion, equality of access, and non-discriminatory provision of evidencebased treatments should be on the forefront of such organisational objectives. 


\section{Limitations and Suggestions for Future Research}

Certain service user groups, such as mixed-race ethnicity, were small, thus affecting the power of the undertaken statistical analysis. For example, one result initially suggested that mixed-race individuals might have been less likely to access treatment. Considering limited number of participants belonging to this patient group, the result may have indicated the presence of type-1-error, which seems to have been the case with the associations initially identified as statistically significant and subsequently disconfirmed after Bonferroni corrections were applied. Hence, the correlations identified prior to Bonferroni adjustments should be interpreted with caution, especially as there is limited rationale for such findings in the context of prior empirical research. As discussed above, statistical literature largely supports the utilisation of Bonferroni method in social and clinical sciences to identify and correct the occurrence of false positives that obscure the outcomes of empirical investigations (Bland and Altman 1995, Mundfrom et al. 2006, Sedgwick 2014).

It seems therefore plausible to suggest that future quantitative research investigating the equality of access to mental healthcare should ideally consider extending the overall recruitment to a larger number of healthcare sites, so that the quantities of participants representing ethnic minorities, including mixed-race individuals, would not inflate the likelihood of type-1-errors occurring in statistical analyses. Likewise, considering the statistically significant disparity identified in the present study, upheld after Bonferroni correction, qualitative research investigating ethnically diverse service users' experiences of inclusiveness in mental healthcare would be of substantial value and provide an understanding of subjective perspectives on equality of access to, and provision of, recommended evidence-based treatments in mental healthcare.

\section{References}

Addington DE, Beck C, Wang J, Adams B, Pryce C, Zhu H, et al. (2010) Predictors of admission in first-episode psychosis: Developing a risk correction model for service comparisons. Psychiatric Services 61(5): 483-488.

Ajnakina O, Lally J, Di Forti M, Kolliakou A, Gardner-Sood P, Lopez-Morinigo J, et al. (2017) Patterns of illness and care over the 5 years following onset of psychosis in different ethnic groups: The GAP-5 study. Social Psychiatry and Psychiatric Epidemiology 52(9): 1101-1111.

Alvarez-Jimenez M, Gleeson JF, Cotton S, Wade D, Gee D, Pearce T, et al. (2009) Predictors of adherence to cognitive-behavioural therapy in first-episode psychosis. The Canadian Journal of Psychiatry 54(10): 710-718.

Anderson KK, Fuhrer R, Schmitz N, Malla AK (2013) Determinants of negative pathways to care and their impact on service disengagement in first-episode psychosis. Social Psychiatry and Psychiatric Epidemiology 48(1): 125-136.

Baba Y, Nemoto T, Tsujino N, Yamaguchi T, Katagiri N, Mizuno M (2017) Stigma toward psychosis and its formulation process: Prejudice and discrimination against early stages of schizophrenia. Comprehensive Psychiatry 73(Feb): 181-186. 
Baker C (2020) Mental health statistics for England: prevalence, services and funding. London, UK: House of Commons Library.

Berry K, Haddock G (2008) The implementation of the NICE guidelines for schizophrenia. Barriers to the implementation of psychological interventions and recommendations for the future. Psychology and Psychotherapy: Theory, Research and Practice 81(4): 419-436.

Bland JM, Altman DG (1995) Multiple significance tests: the Bonferroni method. The BMJ 310(Jan): 170.

Bourque F, van der Ven E, Malla A (2011) A meta-analysis of the risk for psychotic disorders among first- and second-generation immigrants. Psychological Medicine 41(5): 897-910.

Boydell J, Os J van, McKenzie K, Allardyce J, Goel R, McCreadie RG, et al. (2001) Incidence of schizophrenia in ethnic minorities in London: Ecological study into interactions with environment. The BMJ 323(Dec): 1-4.

Bresnahan M, Begg MD, Brown A, Schaefer C, Sohler N, Insel B, et al. (2007) Race and risk of schizophrenia in a US birth cohort: Another example of health disparity? International Journal of Epidemiology 36(4): 751-758.

British Psychological Society (2007) New ways of working for applied psychologists in health and social care: organising, managing, and leading psychological services. Leicester, UK: British Psychological Society.

Campbell P (2007) Hearing my voice. The Psychologist 20(5): 298-299.

Cantor-Graae E, Selten JP (2005) Schizophrenia and migration: a meta-analysis and review. American Journal of Psychiatry 162(1): 12-24.

Conus P, Lambert M, Cotton S, Bonsack C, McGorry PD, Schimmelmann BG (2010) Rate and predictors of service disengagement in an epidemiological first-episode psychosis cohort. Schizophrenia Research 118(1-3): 256-263.

DaCosta J (2020) Insights for implementing change in healthcare. British Journal of Healthcare Management 26(1): 20-26.

Davies J, Hopkins M, Campisi M, Maggs RG (2012) Developing high relational support services for individuals with long term mental health needs: scheme description and service evaluation. Mental Health and Social Inclusion 16(1): 31-40.

Department of Health - DOH (2007) Capabilities for inclusive practice. London, UK: $\mathrm{DOH}$.

Dew SE, Bickman L (2005) Client expectancies about therapy. Mental Health Services Research 7(1): 21-33.

Dominy M, Hayward-Butcher T (2012) Is work good for you? Does paid employment produce positive social capital returns for people with severe and enduring mental health conditions? Mental Health and Social Inclusion 16(1): 14-25.

Farhall J, Freeman NC, Shawyer F, Trauer T (2009) An effectiveness trial of cognitive behaviour therapy in a representative sample of outpatients with psychosis. British Journal of Clinical Psychology 48(Pt 1): 47-62.

Fearon P, Kirkbride JB, Morgan C, Dazzan P, Morgan KD, Lloyd T, et al. (2006) Incidence of schizophrenia and other psychoses in ethnic minority groups: results from the MRC AESOP Study. Psychological Medicine 36(11): 1541-1550.

Fernandes MA, Branco GSC, Costa AS, Silva JS, Feitosa CDA (2017) The relationship between mental health and work: Integrated mental health outpatient centre study. Journal of Nursing 11(10): 4191-4198.

Gaynor K, Dooley B, Lawlor E, Lawoyin R (2014) Predicting uptake of group CBT in a first-episode psychosis cohort. Cognitive Behaviour Therapy 43(2): 105-110. 
Haddock G, Lewis S, Bentall R, Dunn G, Drake R, Tarrier N (2006) Influence of age on outcome of psychological treatments in first-episode psychosis. The British Journal of Psychiatry 188(3): 250-254.

Hansen H, Hjielen S, Davidson L, Moltu C, Veseth M (2018) How do people experience early intervention services for Psychosis? A meta-synthesis. Qualitative Health Research 28(2): 259-272.

Hazell CM, Hayward M, Cavanagh K, Strauss C (2016) A systematic review and metaanalysis of low intensity CBT for psychosis. Clinical Psychology Review 45(Apr): 183-192.

Heckers S, Barch DM, Bustillo J, Gaebel W, Gur R, Malaspina D, et al. (2013) Structure of the psychotic disorders classification in DSM 5. Schizophrenia Research 150(1): $11-14$.

IBM Corp (2017) IBM SPSS Statistics for Windows: Version 25.0. Armonk, NY: IBM Corp.

Jaeger S, Pfiffner C, Weiser P, Längle G, Croissant D, Schepp W, et al. (2013) Long-term effects of involuntary hospitalization on medication adherence, treatment engagement and perception of coercion. Social Psychiatry and Psychiatric Epidemiology 48(11): 1787-1796.

Janssen I, Hanssen M, Bak M, Bijl RV, de Graaf R, Vollebergh W, et al. (2003) Discrimination and delusional ideation. British Journal of Psychiatry 182(1): 71-76.

Karlsen S, Nazroo JY, Mckenzie K, Bhui K, Weich S (2005) Racism, psychosis and common mental disorder among ethnic minority groups in England. Psychological Medicine 35(12): 1795-1803.

Lawlor C, Sharma B, Khondoker M, Peters E, Kuipers E, Johns L (2017) Service user satisfaction with cognitive behavioural therapy for psychosis: associations with therapy outcomes and perceptions of the therapist. British Journal of Clinical Psychology 56(1): 84-102.

Liebermann JA, Stroup TS, McEvoy JP, Swartz MS, Rosenheck RA, Perkins DO, et al. (2005) Effectiveness of antipsychotic drugs in patients with chronic schizophrenia. The New England Journal of Medicine 353(12): 1209-1223.

Lingwood L (2006) Building bridges to social inclusion. In C Jackson, K Hill (eds.), Mental Health Today: A Handbook, 21-27. Brighton, United Kingdom: Pavilion Publishing.

Mankiewicz PD (2013) Cognitive behavioural symptom-oriented understanding of psychosis: abandoning the disease paradigm of schizophrenia. Counselling Psychology Review 28(Mar): 53-63.

Mankiewicz PD (2015) Toward positive psychosocial practice in psychosis: in pursuit of subjective wellbeing in severe mental ill-health. International Journal of Wellbeing 5(4): 120-134.

Mankiewicz PD (2019a) Cognitive restructuring and graded behavioural exposure for persecutory paranoia and agoraphobic anxiety in complex psychosis. Clinical Case Studies 18(2): 143-158.

Mankiewicz PD (2019b) Increasing subjective wellbeing with strengths-based cognitive behavioural psychotherapy in first episode psychosis. International Journal of Happiness and Development 5(3): 183-200.

Mankiewicz PD, Turner C (2012) Do assertive outreach clients with experiences of psychosis receive the NICE recommended cognitive behavioural interventions? An audit. Clinical Psychology Forum 240: 32-37.

Mankiewicz PD, Turner C (2014) Cognitive restructuring and graded behavioural exposure for delusional appraisals of auditory hallucinations and comorbid anxiety in paranoid schizophrenia. Case Reports in Psychiatry 14(2): 1-8. 
Mankiewicz PD, O'Leary J, Collier O (2018) That hour served me better than any hour I have ever had before: service users' experiences of CBTp in first episode psychosis. Counselling Psychology Review 33(Dec): 4-16.

Marshall M, Lewis S, Lockwood A, Drake R, Jones P, Croudace T (2005) Association between duration of untreated psychosis and outcome in cohorts of first-episode patients: a systematic review. Archives of General Psychiatry 62(9): 975-983.

Maura J, Weisman AM (2017) Mental health disparities, treatment engagement, and attrition among racial/ethnic minorities with severe mental illness: A review. Journal of Clinical Psychology in Medical Settings 24(3): 187-210.

McDaid D, Park AL, Iemmi V, Adelaja B, Knapp M (2016) Growth in the use of early intervention for psychosis services: an opportunity to promote recovery amid concerns on health care sustainability. London, UK: Personal Social Services Research Unit.

McManus S, Bebbington P, Jenkins R, Brugha T (Eds.) (2016) Mental health and wellbeing in England: adult psychiatric morbidity survey 2014. Leeds, UK: NHS Digital.

Milan S, Keiley MK (2000) Biracial youth and families in therapy: issues and interventions. Journal of Marital and Family Therapy 26(3): 305-315.

Miles H, Peters E, Kuipers E (2007) Service-user satisfaction with CBT for psychosis. Behavioural and Cognitive Psychotherapy 35(1): 109-116.

Morrison AP, Turkington D, Pyle M, Spencer H, Brabban A, Dunn G, et al. (2014) Cognitive therapy for people with schizophrenia spectrum disorders not taking antipsychotic drugs: a single-blind randomised controlled trial. The Lancet 383(9926): 1395-1403.

Mundfrom DJ, Perrett JJ, Schaffer J, Piccone A, Roozeboom M (2006) Bonferroni adjustments in tests for regression coefficients. Multiple Linear Regression Viewpoints 32(1): $1-6$.

Myhr G, Talbot J, Annable L, Pinard G (2007) Suitability for short-term cognitivebehavioral therapy. Journal of Cognitive Psychotherapy: An International Quarterly 21(4): 334-345.

National Institute for Health and Care Excellence - NICE (2014) Psychosis and schizophrenia in adults: treatment and management. London, UK: NICE.

National Institute for Health and Care Excellence - NICE (2016) Implementing the early intervention in psychosis access and waiting time standard: guidance. London, UK: NICE.

National Social Inclusion Programme (2009) Vision and progress: social inclusion and mental health. London, UK: National Social Inclusion Programme.

Newson JJ, Hunter D, Thiagarajan TC (2020) The heterogeneity of mental health assessment. Frontiers in Psychiatry 11(Feb): 1-24.

Nose M, Barbui C, Tansella M (2003) How often do patients with psychosis fail to adhere to treatment programmes? A systematic review. Psychological Medicine 33(7): 1149-1160.

Pearce J, Rafiq S, Simpson J, Varese F (2019) Perceived discrimination and psychosis: a systematic review of the literature. Social Psychiatry and Psychiatric Epidemiology 54(9): 1023-1044.

Rajamanickam R, Zahir MZM, Zainudin TNAT, Rahman ZA (2019) Mental health legislation for the elderly with mental health problems. The European Proceedings of Social and Behavioural Sciences 70(11): 95-102.

Royal College of Psychiatrists - RCP (2009) Mental health and social inclusion: making psychiatry and mental health services fit for the $21^{\text {st }}$ century. London, UK: RCP. 
Royal College of Psychiatrists - RCP (2019) National clinical audit of psychosis: national report for the early intervention in psychosis spotlight audit 2018/2019. London, UK: Healthcare Quality Improvement Partnership.

Sedgwick P (2014) Multiple hypothesis testing and Bonferroni's correction. The BMJ 349(Oct): 1-3.

Simon GE, Coleman KJ, Yarborough BJ, Operskalski B, Stewart C, Hunkeler E, et al. (2017) Incidence and presentation of first-episode psychosis in a population-based sample. Psychiatric Services 68(5): 456-461.

Slade T, Johnston A, Oakley-Browne MA, Andrews G, Whiteford H (2009) National survey of mental health and wellbeing: methods and key findings. Australian and New Zealand Journal of Psychiatry 43(7): 594-605.

Social Exclusion Unit - SEU (2004) Mental health and social exclusion. London, UK: Office of the Deputy Prime Minister.

Stewart KD (2013) Factors contributing to engagement during the initial stages of treatment for psychosis. Qualitative Health Research 23(3): 336-347.

Stowkowy J, Addington D, Liu L, Hollowell B, Addington J (2012) Predictors of disengagement from treatment in an early psychosis program. Schizophrenia Research 136(1-3): 7-12.

Sturgis P, Brunton-Smith I, Kuha J, Jackson J (2014) Ethnic diversity, segregation and the social cohesion of neighbourhoods in London. Ethnic and Racial Studies 37(8): 1286-1309.

Veling W, Selten JP, Susser E, Laan W, Mackenbach JP, Hoek HW (2007) Discrimination and the incidence of psychotic disorders among ethnic minorities in the Netherlands. International Journal of Epidemiology 36(4): 761-768.

Wade D, Harrigan S, Harris MG, Edwards J, McGorry PD (2006) Pattern and correlates of inpatient admission during the initial acute phase of first-episode psychosis. Australian and New Zealand Journal of Psychiatry 40(5): 429-436. 
